\title{
Xylanase inhibitors bind to non-starch polysaccharides
}

\author{
Ellen Fierens, Kurt Gebruers", Christophe M. Courtin and Jan A. Delcour
}

Laboratory of Food Chemistry and Biochemistry, Department of microbial and molecular systems, Katholieke Universiteit Leuven, Kasteelpark Arenberg 20, Box 2463, 3001 Leuven, Belgium

*Corresponding author: Tel.: +32(0)16321634, Fax: +32(0)16321997

e-mail of all authors : Ellen.fierens@,biw.kuleuven.be

Kurt.gebruers@biw.kuleuven.be

Christophe.courtin@,biw.kuleuven.be

Jan.delcour@biw.kuleuven.be 


\section{Abstract}

2 This study is an in-depth investigation of the interaction between polysaccharides and the 3 proteinaceous xylanase inhibitors, Triticum aestivum xylanase inhibitor (TAXI), xylanase

4 inhibitor protein (XIP) and thaumatin-like xylanase inhibitor (TLXI). The binding affinities of all

5 three known types of xylanase inhibitors from wheat are studied by measuring the residual

6 xylanase inhibition activity after incubation of the inhibitors in the presence of different

7 polysaccharides, such as $\beta$-glucans and (arabino)xylans. The binding affinities of all three

8 xylanase inhibitors for (arabino)xylans increased with decreasing arabinose to xylose ratio (A/X-

9 ratio). This phenomenon was observed both with water-extractable and water-unextractable

10 (arabino)xylans. The inhibitors also interacted with different soluble and insoluble $\beta$-glucans.

11 None of the inhibitors tested had the ability to hydrolyze the polysaccharides investigated. The

12 present findings contribute to the unraveling of the function of xylanase inhibitors in nature and

13 to the prediction of the effect of added xylanases in cereal based biotechnological processes, 14 such as bread making and gluten-starch separation.

16 Keywords: wheat, xylanase inhibitors, $\beta$-glucan, xylan, binding, polysaccharide 
19 In the last decade, the occurrence of three types of xylanase inhibitors in cereals has been

20 demonstrated, i.e. Triticum aestivum xylanase inhibitor (TAXI), xylanase inhibitor protein (XIP)

21 and thaumatin-like xylanase inhibitor (TLXI) (1-3). The three types have distinct structures and

22 differ in their specificities towards endo-beta-1,4-xylanases, further referred to as xylanases.

23 They significantly impact the functionalities of a number of xylanases in cereal based

24 biotechnological applications, such as bread making $(4,5)$ and gluten-starch separation $(6)$.

25 The functions of these inhibitor proteins in the plant are not yet understood. Their lack of 26 inhibition activity against endogenous xylanases disaffirms a regulatory role in plant

27 development $(3,7,8)$. However, based on their distinct specificities towards xylanases of 28 microbial origin, the ability of TAXI to inhibit two xylanases of glycoside hydrolase family 11 of 29 the cereal pathogen Fusarium graminearum (9), the fact that some TAXI and XIP genes are 30 induced by pathogens and wounding $(10,11)$ and the homologies of TLXI and XIP with 31 pathogenesis-related proteins of family 5 (PR-5) and 8 (PR-8), respectively, they are believed to 32 be involved in plant defense. PR proteins, which have been classified as a major group of 33 defense-related proteins, are proteins encoded by the host plant but induced in pathological or 34 other stress situations. To date, PR proteins have been classified into 17 structurally and 35 functionally distinct families (12-14), some of which consist of proteins with known biological 36 functions.

37 The binding of XIP to polysaccharides has been reported. More exactly, XIP binds to Blue starch 38 and wheat arabinoxylan but not to cellulose and chitin (15). Rouau and coworkers (16) reported 39 that XIP binds to water-unextractable arabinoxylan (WUAX) and not to water-extractable 40 arabinoxylan (WEAX). XIP loses its inhibition activity upon binding, in their view, resulting in a 
41 strongly depressed inhibition activity in the vicinity of WUAX. In contrast, in the vicinity of

42 WEAX, the XIP activity is not affected. The xylanase is therefore proportionally more active on

43 WUAX (arabinoxylan solubilisation) than on WEAX (arabinoxylan depolymerisation). The net

44 effect is an increase in the soluble high molecular weight arabinoxylan levels, which are

45 beneficial for dough properties, and a larger decrease in WUAX levels, which are detrimental to

46 both dough and bread properties (17). The above opens perspectives for improving enzyme

47 performance in bread making, by steering the xylanase selectivity by a specific inhibitor.

48 In addition, insight in the binding of the xylanase inhibitors to polysaccharides can contribute to

49 our understanding of their functions in plants. After all, for the PR-5 protein family, binding to

50 polysaccharides has been reported to be involved in their antifungal mechanism. Members of this

51 family are also called the thaumatin-like proteins (TLPs) because they are all homologues to

52 thaumatin, a sweet tasting protein found in the arils of fruits of the African shrub

53 Thaumatococcus daniellii (18). Most TLPs are believed to be involved in plant defense because

54 their synthesis is induced upon challenge of the plant by pathogens or exposure to abiotic stress

55 factors (e.g. drought). In the last two decades, efforts have been directed towards unraveling the

56 exact physiological role of TLPs. For several TLPs, in vitro antifungal activity has been

57 discovered. Experiments with transgenic wheat and rice plants showed that TLPs enhance

58 resistance against various pathogens $(19,20)$. However, the underlying mechanism is still not

59 fully understood. Some TLPs function by dramatically increasing microbial membrane

60 permeability (21) and $\beta$-glucan binding. Also, several TLPs possess hydrolyzing activity (22-25).

61 In the light of the importance of the interaction of xylanase inhibitors with arabinoxylan and $\beta$ -

62 glucan from a biotechnological as well as a plant physiological point of view, a thorough

63 investigation of the polysaccharide binding and hydrolyzing properties (if any) of the three 
64 different xylanase inhibitors from wheat is important. After all, Rouau et al. (16) tested the

65 binding of XIP for only a few polysaccharides. The fact that TLXI is homologous to the above

66 cited TLPs further indicates the need for this investigation. In this context, the present report

67 focuses on the affinity of all three types of xylanase inhibitors for both $\beta$-glucans and 68 (arabino)xylans and whether the inhibitors can hydrolyze these polysaccharides.

\section{Materials and Methods}

\section{$70 \quad$ Materials}

71 Wheat (cultivar Soissons, AVEVE, Landen, Belgium) whole meal was prepared using a

72 Cyclotec 1093 sample mill with a $1.0 \mathrm{~mm}$ sieve (Tecator, Hogänäs, Sweden). All electrophoresis

73 media and molecular mass markers were from GE Healthcare (Uppsala, Sweden), unless

74 specified otherwise. Glycoside hydrolase family 11 xylanase (pI 5.5) from Trichoderma

75 longibrachiatum (also known as T. reesei), i.e. Xyn I (NCBI accession number CAA49294),

76 Xylazyme AX tablets, azo-barley glucan, azo-wheat AX, CM-pachyman and oat $\beta$-glucan were

77 from Megazyme (Bray, Ireland). Birchwood xylan, oat spelt arabinoxylan, xylose, zymosan

78 (consisting of protein-carbohydrate complexes from the yeast cell wall of Saccharomyces

79 cerevisiae), paramylon, cellulose, pullulan, laminarin, bovine serum albumin (BSA) and all other

80 chemicals were from Sigma-Aldrich (Bornem, Belgium). Pustulan was from Calbiochem

81 (Darmstadt, Germany). Water soluble birchwood xylan and oat spelt arabinoxylan were prepared

82 as described by He et al. (26). Native wheat WE-AX and WU-AX were prepared as described by

83 Moers et al. (27). The insoluble polysacharides were washed by suspending the polysaccharide

$84(0.5 \mathrm{~g})$ in water $(50.0 \mathrm{ml})$, shaking this supension for $60 \mathrm{~min}$ and centrifugating $(15000 \mathrm{~g}, 10$ 
$\left.85 \min , 20^{\circ} \mathrm{C}\right)$. The supernatant was removed and this step was repeated twice with the precipitate.

86 The precipitate was freeze-dried before use in the assays.

87 Purification of xylanase inhibitors from wheat whole meal

88 TAXI and XIP were purified from wheat whole meal as described earlier (28) as was TLXI (3).

\section{Protein content determination}

90 Protein concentrations were determined by the Bradford Coomassie brilliant blue method with

91 BSA as standard (29). For pure TAXI, XIP and TLXI samples, protein concentrations were

92 determined by extinction measurements at $280 \mathrm{~nm}$ using specific absorbance values of 0.788 ,

932.442 and $1.457 \mathrm{AU}$ for $1.0 \mathrm{mg} / \mathrm{ml}$ TAXI, XIP and TLXI, respectively $(1.000 \mathrm{~cm}$ UV-cell path 94 length).

\section{Xylanase inhibition assay (Xylazyme $\mathrm{AX}$ method)}

96 Inhibition activities were determined with the colorimetric Xylazyme AX method (28). The

97 xylanase solution was prepared in sodium acetate buffer $(25 \mathrm{mM}, \mathrm{pH} 5.0)$ with BSA $(0.5 \mathrm{mg} / \mathrm{ml})$

98 and contained 2.0 xylanase units per ml. Xylanase units were defined as described by Gebruers

99 et al. (28). Under the conditions of the assay, the xylanase concentration corresponding to 1.0

100 unit Xyn I was approximately $8.9 \mathrm{nM}$. All measurements were performed in triplicate.

101 Electrophoresis

102 Regular SDS-PAGE

103 SDS-PAGE under non-reducing and reducing conditions was performed on $20 \%$ 104 polyacrylamide gels with a PhastSystem unit, as described in the GE Healthcare separation 105 technique file 110. 2-mercapto-ethanol $[5.0 \%(\mathrm{v} / \mathrm{v})]$ was added to the sample buffer as reducing 
106 agent. All gels were stained with silver in accordance with the instructions of the manufacturer of

107 the gels (development technique file no. 210).

108 Substrate SDS-PAGE

109 Substrate SDS-PAGE for the detection of xylanases was based on protocols by Biely et al. (30),

110 Schwarz et al. (31) and Royer and Nakas (32), using a mini-Protean-3-system (Biorad, Nazareth,

111 Belgium) with slab gels $(140.0 \mathrm{~mm} \times 160 \mathrm{~mm} \times 1.5 \mathrm{~mm})$ consisting of a stacking gel (4\%

112 polyacrylamide) on top of a running gel (12\% polyacrylamide). The latter contained $0.28 \%$

$113(\mathrm{w} / \mathrm{v})$ azo-wheat arabinoxylan or azo-barley $\beta$-glucan as substrate. Separation was with a current

114 of $20 \mathrm{~mA}$ per gel for 60 min (Power-Pac-200 power supply, Biorad). After separation, the gels

115 were washed in $50 \%(\mathrm{v} / \mathrm{v})$ ethanol for 30 minutes and incubated in sodium acetate buffer (25

$116 \mathrm{mM}, \mathrm{pH} 5.0)$ at $37^{\circ} \mathrm{C}$ until hydrolysis zones became visible.

\section{Analysis of the binding of xylanase inhibitors to polysaccharides}

118 Glucans and insoluble (arabino)xylans

119 To avoid the unspecific binding of the xylanase inhibitor to the polysaccharides, casein and

120 Tween 20 were used as blocking agents. The polysaccharides $(5.0 \mathrm{mg})$ were suspended in

121 casein/Tween solution [0.5 ml; $0.1 \%(\mathrm{w} / \mathrm{v})$ casein with $0.02 \%(\mathrm{v} / \mathrm{v})$ Tween in $25 \mathrm{mM}$ sodium

122 acetate buffer $\mathrm{pH}$ 5.0]. These suspensions were shaken at room temperature for $60 \mathrm{~min}$.

123 Subsequently, the inhibitor solution $(200 \mu \mathrm{l} ; 1.6 \mu \mathrm{M}$ in $25 \mathrm{mM}$ sodium acetate buffer, $\mathrm{pH} 5.0)$

124 was added and the mixture was again incubated for $60 \mathrm{~min}$ at room temperature. The

125 polysaccharides were removed by centrifugation (15000 g, $10 \mathrm{~min}$, room temperature) and the

126 inhibition activity against Xyn I of the resulting supernatant (100 $\mu 1)$ was tested with the

127 Xylazyme AX method (cfr. supra). Comparison with the inhibition activity of the reference

128 sample, incubated in the absence of polysaccharides, allowed calculation of the fraction of 
129 inhibitor inactivated by interaction with the polysaccharides. This fraction is defined as the

130 inhibitor bound to the polysaccharide. All measurements were performed in triplicate.

131 In addition, samples of the supernatant and the precipitate were analyzed on SDS-PAGE. To this

132 end, the precipitate was washed three times by the addition of sodium acetate buffer $(1.0 \mathrm{ml} ; 25$

$133 \mathrm{mM}, \mathrm{pH} 5.0)$, shaking for $10 \mathrm{~min}$ and subsequent centrifugation $(15000 \mathrm{~g}, 10 \mathrm{~min}$, room

134 temperature). The washed precipitate was then incubated at $100{ }^{\circ} \mathrm{C}$ in a $1: 1$ dilution of SDS-

135 PAGE sample buffer $(0.25 \mathrm{ml} ; 4.0 \%$ SDS, $30 \%$ glycerol, $0.04 \%$ bromofenol blue in $125 \mathrm{mM}$

136 Tris, $\mathrm{pH} 6,8)$ in sodium acetate buffer $(0.25 \mathrm{ml} ; 25 \mathrm{mM}, \mathrm{pH} 5.0)$ and analyzed on regular SDS-

137 PAGE.

138 Soluble (arabino)xylans - First, the inhibitors $(10 \mu \mathrm{l} ; 0.5 \mu \mathrm{M}$ TAXI, $0.2 \mu \mathrm{M}$ XIP or $0.4 \mu \mathrm{M}$

139 TLXI in $25 \mathrm{mM}$ sodium acetate buffer, $\mathrm{pH}$ 5.0) were preincubated with the soluble

140 (arabino)xylan $(55 \mu \mathrm{l} ; 5 \mathrm{mg} / \mathrm{ml}$ in $25 \mathrm{mM}$ in sodium acetate buffer, $\mathrm{pH} 5.0$ ) for $60 \mathrm{~min}$ at room

141 temperature. The samples were then put at $40{ }^{\circ} \mathrm{C}$ for $10 \mathrm{~min}$, after which the reaction was started

142 by the addition of the xylanase solution $(15 \mu 1 ; 0.25 \mu \mathrm{M}$ of xylanase in $25 \mathrm{mM}$ sodium acetate

143 buffer, pH 5.0 containing $1 \mathrm{mg} / \mathrm{ml} \mathrm{BSA}$ ). The samples were incubated for different periods of

144 time up to $20 \mathrm{~min}$ at $40{ }^{\circ} \mathrm{C}$. The reaction was stopped by adding $120 \mu 1$ of dinitrosalicylic acid

145 reagent $(5.0 \mathrm{~g}$ dinitrosalicylic acid and $150.0 \mathrm{~g}$ potassium-sodium-tartrate in $500 \mathrm{ml} 0.4 \mathrm{M}$

$146 \mathrm{NaOH})$ and putting the samples on ice. After incubation at $100{ }^{\circ} \mathrm{C}(5 \mathrm{~min})$ and cooling, the

147 samples were centrifuged for $5 \mathrm{~min}$ at $3000 \mathrm{~g}, 150 \mu \mathrm{l}$ of each sample was transferred to a

148 microtiterplate and the absorbances were measured at $540 \mathrm{~nm}$ against a control (buffer instead of

149 enzyme and inhibitor). The inhibition activities were calculated from the difference in production

150 of reducing sugars between samples with and without inhibitor. The level of inhibitor bound to

151 the soluble substrate was determined by comparing this activity to the inhibition activity 
152 obtained when the inhibitor and enzyme were preincubated and the soluble (arabino)xylan was

153 added in a final step to start the reaction. All measurements were performed in triplicate.

\section{Analysis of the hydrolysis of polysaccharides by xylanase inhibitors}

155 The polysaccharides $(5.0 \mathrm{mg})$ were suspended in the xylanase inhibitor solutions $(1.0 \mathrm{ml}$; up to $1562.6 \mu \mathrm{M}$ inhibitor in $25 \mathrm{mM}$ sodium acetate buffer, $\mathrm{pH} 5.0$ containing $0.05 \%$ sodium azide).

157 These suspensions were incubated at $40{ }^{\circ} \mathrm{C}$ up to $72 \mathrm{~h}$. At different time intervals, samples (125

$158 \mu 1)$ were withdrawn and immediately frozen in liquid nitrogen. At the end of the incubation time,

159 all samples were rapidly defrosted and centrifuged (10000 g, 5 min, room temperature). The

160 concentrations of reducing sugars in the supernatants were measured with tetrazolium blue. To

161 this end, samples $(50 \mu \mathrm{l})$ were mixed with $4.0 \mathrm{ml}$ tetrazolium blue solution $[0.1 \%(\mathrm{w} / \mathrm{v})$

162 tetrazolium blue chloride in $50 \mathrm{mM} \mathrm{NaOH}$ containing $500 \mathrm{mM}$ sodium potassium tartrate (33)].

163 After incubation at $100{ }^{\circ} \mathrm{C}$ for 3 min and cooling, the absorbances were measured at $660 \mathrm{~nm}$

164 against controls containing no inhibitor and which were incubated similarly. Standard curves 165 with glucose and xylose were made for assessing the hydrolysis of glucans and (arabino)xylans, 166 respectively $(0-1.0 \mathrm{mg} / \mathrm{ml})$.

167 Results

168 Binding of the xylanase inhibitors to (arabino) xylans

169 In a first experiment, the binding of TAXI, XIP or TLXI to different insoluble (arabino)xylans

170 was visualised by comparing the SDS-PAGE profiles of the supernatants obtained after the

171 incubation of xylanase inhibitors with insoluble (arabino)xylans with the SDS-PAGE profiles of

172 the control samples. The fractions bound to the (arabino)xylans were equally analyzed. Figure 1

173 shows only the part of the SDS-PAGE gel where the inhibitor proteins are visible. These parts 
174 correspond to $40 \mathrm{kDa}, 30 \mathrm{kDa}$ and $18 \mathrm{kDa}$, for TAXI, XIP and TLXI, respectively. For wheat

175 WUAX, oat spelt arabinoxylan and birchwood xylan, the inhibitors were clearly present in the 176 precipitate obtained after centrifugation and multiple washing steps. This was interpreted as 177 evidence for the binding of TAXI, XIP and TLXI to all three types of (arabino)xylans. Only for 178 TAXI and XIP, incubated with wheat WUAX, there was still inhibitor present in the supernatant. 179 For all other samples, no inhibitor was visible in this fraction, suggesting that all TAXI, XIP and 180 TLXI was bound.

181 To confirm these results the inhibition activities in the supernatants were compared to those in 182 the control samples. First, the interference of additional soluble arabinoxylan in the Xylazyme 183 assay was ruled out. Even when $750 \mu \mathrm{g}$ additional WE-AX was added to the samples no 184 influence was seen on the xylanase activity measured with the Xylazyme tablet. The decrease in 185 xylanase activity measured when the supernatant fraction is added to the assay is thus solely 186 caused by the presence of the inhibitor and not by the solubilisation of part of the insoluble 187 arabinoxylan. The results of the inhibition activities in the supernatant show the same trend as 188 with SDS-PAGE (Figure 2), i.e. all types of inhibitors bound to all insoluble (arabino)xylans. 189 Only for wheat WUAX a significantly lower binding interaction was seen.

190 As Birchwood xylan has no arabinose substituent (A/X-ratio of 0.0 ), oat spelt arabinoxylan has 191 an A/X-ratio of approximately 0.1 , and wheat WUAX has an A/X-ratio of approximately 0.5 , the 192 results seem to indicate that the binding of the xylanase inhibitors to (arabino)xylans depends on 193 the A/X-ratio, with higher A/X-ratios resulting in reduced binding. However, for TLXI, 194 quantitative binding was also observed for wheat WUAX. The relative binding decreased with 195 increasing A/X-ratios. TAXI and XIP showed lower binding affinities for the three 196 (arabino)xylans than TLXI, but a similar trend was seen. 
197 To assess the binding affinity of TAXI, XIP and TLXI for soluble (arabino)xylans, the inhibitors

198 were preincubated with either xylanase or (arabino)xylan for $60 \mathrm{~min}$ at room temperature. The

199 reaction was then started by adding the polysaccharide or xylanase, respectively. The increase in

200 the reducing sugar levels was measured for $20 \mathrm{~min}$. Figure 3 shows a typical progress curve

201 obtained in this manner. The inhibition activities were calculated from the differences in the rate

202 of the release of reducing sugars. All three inhibitors were partially inactivated, probably by

203 being bound to the soluble (arabino)xylans (Figure 4). However, the trends were different from

204 those seen for their insoluble counterparts. Under the experimental conditions, the binding of

205 TLXI was still most prominent, but, in contrast to the insoluble (arabino)xylans, the binding of

206 TAXI was somewhat higher than that of XIP. The differences between the different

207 (arabino)xylans were less pronounced. The levels of the binding to birchwood xylan were still

208 the highest, but there was little if any difference between binding to oat spelt and wheat WEAX.

\section{Binding of the xylanase inhibitors to glucans}

210 Table 1 presents the differences in structure and solubility of the glucans used in present study.

211 The SDS-PAGE profiles of the inhibitor fraction either bound or not bound to the insoluble

212 glucans were compared to those of the control inhibitor samples (Figure 5). In contrast to what

213 was observed with (arabino)xylans, in this case the inhibitors were present in both the

214 supernatant and the precipitate for almost all glucans tested. For some of the glucans, hardly any

215 binding was observed. Zymosan, in contrast, left no inhibitor in the supernatant. In general,

216 TLXI had the highest glucan binding affinity.

217 Much as done for the insoluble (arabino)xylans, a more accurate assessment was made by 218 comparing the inhibition activities in the supernatants after incubation of the xylanase inhibitors 219 with insoluble glucans with those of the control inhibitor samples (Figure 6). For the three types 
220 of inhibitors, a high binding affinity for zymosan was observed. TLXI also bound to paramylon

221 and, to a lesser extent, to oat $\beta$-glucan. In contrast to the high binding to zymosan, low binding

222 percentages were seen for the binding of XIP to oat $\beta$-glucan and pustulan and for the binding of

223 TAXI to paramylon and cellulose.

224 Comparison of the above with the results obtained for the soluble glucans (Figure 7) revealed 225 that, in contrast to the insoluble $\beta$-(1,3)(1,6)-glucan (zymosan), no binding to the soluble $\beta$ 226 (1,3)(1,6)-glucan (laminarin) was detected. The soluble $\beta$-(1,3)-glucan, i.e. CM-pachyman, 227 bound with TAXI and TLXI and, to a smaller extent, with XIP. Note that the insoluble $\beta-(1,3)-$ 228 glucan (paramylon), did not bind to XIP. The binding for TAXI was lower than that for TLXI.

229 Only XIP was able to bind to the $\alpha-(1,4)(1,6)$-glucan (pullulan).

\section{Hydrolysis of non-starch polysaccharides by the xylanase inhibitors}

231 The ability of the inhibitors to hydrolyze (arabino)xylans and $\beta$-glucans was studied. The same

232 polysaccharides used in the above binding experiments were tested with the same three xylanase

233 inhibitors from wheat. None of the polysaccharide-inhibitor combinations resulted in detectable

234 hydrolysis after $72 \mathrm{~h}$ of incubation. In the substrate gels with incorporated azo-wheat 235 arabinoxylan or azo-barley- $\beta$-glucan, the xylanase inhibitors samples caused no hydrolysis spot 236 became visible (results not shown).

\section{Discussion}

\section{Interaction of the xylanase inhibitors with polysaccharides}

240 The binding of XIP to wheat arabinoxylan has been described before. Sancho and co-workers 241 (15) reported XIP to bind to WUAX. In SDS-PAGE, they observed that XIP disappeared from 
242 the liquid phase after incubation with the insoluble non-starch polysaccharide. Rouau et al. (16)

243 performed a more quantitative measurement of the binding of XIP to wheat arabinoxylan and

244 made a distinction between WEAX and WUAX. They observed only a slight reduction of the

245 inhibition activity in the presence of WEAX, while, in the presence of a crude cell wall material

246 preparation [mainly composed of WUAX (53\%)] a strong reduction in inhibition activity was

247 seen.

248 Our study not only confirmed these results but, in addition, demonstrated that the ability to bind 249 (arabino)xylans is not restricted to XIP, but that TAXI and TLXI are also able to interact. For the 250 three inhibitors, a similar trend was seen, i.e. the affinity increased with decreasing $\mathrm{A} / \mathrm{X}$-ratio.

251 This trend was most clear with the insoluble (arabino)xylans. This dependency on A/X-ratio 252 suggested that the interaction between the inhibitor and the xylan chain was more outspoken 253 when no substituents are present. The highest level of binding was observed with TLXI. For XIP, 254 the affinity for the insoluble polysaccharides was much higher than for their soluble counterparts.

255 Though the ratios of inhibitor to polysaccharide used in the present study were in the same order 256 of magnitude as the ones used by Rouau et al. (16), the differences between the binding of XIP to 257 WE-AX and WU-AX were less pronounced in the present study. This is probably due to the use 258 of crude wheat cell wall material by Rouau et al. (16) instead of purified WU-AX. The crude cell 259 wall material contains WUAX with a different structure than the one used in the present study 260 and it may contain other constituents which interfere with the interaction with XIP. It should be 261 noted that the binding of TAXI to wheat arabinoxylan seems to be independent of the solubility 262 of this polysaccharide, but that it was higher for the insoluble oat spelt arabinoxylan and the 263 insoluble birchwood xylan than for their soluble counterparts. TAXI and XIP had comparable 264 affinities for (arabino)xylans. 
265 Sancho et al. (15) studied the interaction between XIP and starch (Blue Starch), cellulose and 266 chitin. While XIP was able to interact with starch, no interaction with XIP was observed for

267 chitin and cellulose. Our results were in line with the latter, as also here no interaction between 268 XIP and cellulose could be detected. However, after incubation with pullulan, a linear $\alpha$ 269 (1,4)(1,6)-glucan, a decrease in inhibition activity was measured, suggesting an interaction 270 between XIP and this polysaccharide.

271 A number of reports describe the interaction of TLPs with $\beta$-(1,3)-glucans. Trudel et al. (22)

272 observed that three barley TLPs bind to $\beta$-(1,3)-glucans independent of the presence of $\beta-(1,6)$

273 linkages. TLP binding polysaccharides included paramylon, zymosan, pachyman and laminarin.

274 The authors did not detect binding to $\beta-(1,3)(1,4)-, \beta-(1,4)$ - and $\beta-(1,6)$-glucans, like lichenan, 275 cellulose and pustulan, respectively. In line with the above, Osmond et al. (24) did not observe 276 any adsorption of barley PR-5 proteins to chitin, pustulan or cellulose. The observed adsorption 277 to pachyman and curdlan suggested a preference for polysaccharides that predominantly contain 278 linear $\beta-(1,3)$-glucans.

279 The results obtained here for TLXI, a wheat TLP, demonstrated a significant level of binding to 280 zymosan and no binding at all to laminarin, two glucans with $\beta-(1,3)(1,6)$-bonds. It may be that 281 the interaction between TLXI and zymosan was non-specific and that the binding to this 282 preparation was caused by impurities. After all, the zymosan samples used consisted of almost 283 the entire yeast cell wall of Saccharomyces cerevisiae. The results shown allow concluding that 284 TLXI binds to both soluble and insoluble $\beta-(1,3)$-glucans and to a lesser extent to insoluble $\beta$ 285 (1,3)(1,4)-glucans. For TAXI, low binding to soluble and insoluble $\beta$-(1,3)-glucans and insoluble $286 \beta$-(1,4)-glucans was seen, while XIP bound to insoluble $\beta$-(1,6)- and $\beta-(1,3)(1,4)$-glucan and to 287 soluble $\beta$-(1,3)-glucan. 
289 Based on their homologies with PRs, hypotheses can be formulated on the binding sites 290 responsible for the interaction with non-starch polysaccharides, for XIP and TLXI.

291 XIP shows structural homology to chitinases of class III, which belong to the group of PR-8 292 proteins. One of the major latex proteins, (Hevea brasiliensis) hevamine, displays lysozyme and 293 chitinase activities (34). The structure of hevamine is similar to that of a bacterial chitinase from 294 Glycoside hydrolase family 18 (35). Chitinases may protect the seed against the invasion by 295 chitin-containing pathogenic fungi because the substrates for chitinase are found in some fungal 296 cell walls, but not in plants. For XIP, however, no chitinase activity was observed (36). One of 297 the two catalytic glutamic acid residues of chitinases is nevertheless conserved in the structure of 298 XIP (Glu128 XIP) but its side chain is fully engaged in salt bridges with two neighboring arginine

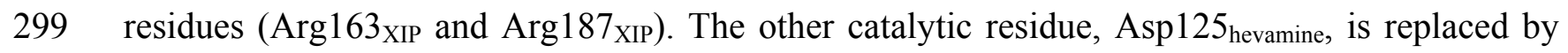

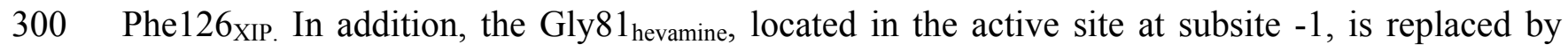
301 Tyr80 XIP (36). This tyrosine side chain fills the subsite area and makes a strong hydrogen bond 302 with the side chain of the Glu190 XIP located at the opposite site of the cleft, preventing access of 303 a polysaccharide to the catalytic glutamic acid. However, three aromatic residues $\left(\operatorname{Trp} 255_{\text {hevamine, }}\right.$ 304 Tyr $183_{\text {hevamine }}$ and Tyr6 $\left.6_{\text {hevamine }}\right)$ present in the active centre of hevamine are conserved in the 305 XIP-structure and correspond to Trp256 XIP, Tyr189 XIP and Phe11 XIP $(36)$. The aromatic side 306 chains of Trp255 hevamine and Tyr6 $6_{\text {hevamine }}$ stack against the hydrophobic face of chitin-backbone.

307 The hydroxyl group of Tyr $183_{\text {hevamine }}$ is within hydrogen-bonding distance of the -1 sugar residue 308 of the substrate and contributes to the catalysis. Since these residues are conserved in the XIP309 structure, it is very well possible that they are responsible for the interaction between XIP and 310 polysaccharides. In addition to these three aromatic residues, there are several other residues 
311 causing hydrogen-bonding contacts in the cleft of hevamine (atoms of the main chain of 5

312 residues and atoms of the side chain of 3 residues). In the corresponding cleft in the XIP

313 structure, the side chains of the corresponding residues show similar conformations (36).

314 For TLXI, a TLP, a similar exercise can be made. Indeed, for several TLPs the crystal structure

315 has been resolved. Osmond et al. (24) compared the amino acid sequences of two barley TLPs,

316 HvPR5b and HvPR5c. Although their sequences show $60 \%$ homology, they largely differ in

317 their binding activity. While HvPR5c interacts tightly with $\beta$-glucans, the interaction for

318 HvPR5b is only weak. Both proteins consist of a flattened $\beta$-barrel adjacent to a more flexible

319 region rich in loops and coils which are stabilized by disulfide bonds. A large cleft is present

320 between these two regions. Calculations of the electrostatic potentials at the surfaces of the

321 proteins revealed a concentration of acidic amino acid residues in the cleft. This negatively

322 charged cleft is thought to be the binding site for polysaccharides. The lack of binding of

323 HvPR5b is likely to be explained by the absence of several amino acid residues along the cleft

324 that might form hydrogen bonds with the bound $\beta$-glucans. In contrast to these two proteins,

325 TLXI belongs to the group of smaller PR-5 proteins, which are mainly found in cereals (37).

326 These smaller TLPs miss the more flexible region, rich in loops and coils. Hence, the negatively

327 charged cleft is not as distinctive as for the larger TLPs. Reiss et al. (37) describe four of these

328 smaller TLPs from barley. TLXI shows the highest homology to TLP3. TLP3 clearly differs

329 from the other small barley TLPs (TLP1, 2 and 4), in that it lacks the acidic residues (Glu83 ${ }_{\text {TLP1, }}$

330 Asp93 $\left.{ }_{\mathrm{TLP} 1}, \mathrm{Asp} 101_{\mathrm{TLP} 1}\right)$ present at identical positions in its structure. In TLP3, they are replaced

331 by Gln87 8 TLP3, Tyr95 $5_{\mathrm{TLP} 3}$, Lys $103_{\mathrm{TLP} 3}$, respectively. In contrast to what is the case for TLP3, one

332 of the acidic residues is conserved (Glu87 ${ }_{\mathrm{TLXI}}$ ) in TLXI, but the two others are identical to those

333 of TLP3 (Tyr96 ${ }_{\mathrm{TLXI}}$ and Lys105 ${ }_{\mathrm{TLXI}}$ ). According to Reiss and coworkers (37) these residues 
334 might form the binding site for ligands unidentified at present, possibly $\beta$-glucans. In this view,

335 the differences between the small barley TLPs might be an indication for distinct substrate

336 binding specificities between these proteins.

337 For TAXI, it is more difficult to bring forward a hypothesis on the binding sites responsible for

338 the interaction with non-starch polysaccharides, as it shows homology to aspartic proteases and

339 not to polysaccharide binding proteins. However, non-polar and hydrogen-bond interactions are

340 involved in the binding of proteins to polysaccharides. Over $64 \%$ of the TAXI sequence consists

341 of non-polar and over $20 \%$ of uncharged polar residues (38). It is possible that a combination of

342 some of these residues makes up a sugar binding region.

\section{Relevance of the present findings}

344 In the light of the results obtained here, a hypothesis on the relevance of binding of xylanase

345 inhibitors to non-starch polysaccharides in biotechnological processes such as bread making can

346 be brought forward. As Rouau et al. (16) and Sibbesen and Soerensen (39) already suggested, the

347 difference in the binding affinity of the cereal xylanase inhibitors between soluble and insoluble

348 xylans may be an interesting mechanism for steering the enzyme selectivity and improving the

349 biocatalyst's performance in this process.

350 However, it should be noted that the conditions in the in vitro polysaccharide binding 351 experiments are not the same as in bread making.

352 First of all, all in vitro binding experiments were performed on either soluble or insoluble xylan

353 while, in wheat matrices, these two polysaccharide types coexist. Secondly, while in both

354 systems a large excess of polysaccharide is present, the ratio of polysaccharide to inhibitor is

355 approximately ten times higher in the in vitro experiments than that in wheat dough systems.

356 Thirdly, the in vitro experiments were performed in excess of water, while in bread making the 
357 level of free water is limited, which, on the one hand, may well reduce the mobility of the 358 inhibitors because of viscosity effects, but, on the other hand, because of concentration effects, 359 may well increase the probability of successful encounters and, hence, interactions between the 360 molecules involved. Finally, it is of note that in the in vitro experiments there was a 361 preincubation step allowing interaction between the inhibitor and the enzyme or the inhibitor and 362 the substrate, while this is not the case in the bread making process.

363 With regard to their function in the plant, a role in plant defence has been suggested for xylanase 364 inhibitors, though the exact mechanism is not yet understood. Since all three cereal xylanase 365 inhibitors are secreted outside the plant cell $(8,38,40)$ and xylans and $\beta$-glucans occur in the 366 cereal cell wall, the binding of the inhibitors to these polysaccharides may result in a correct 367 localization of the inhibitors to defend the plant cell against invasion of pathogenic fungi. On the 368 other hand, $\beta$-glucans also occur in the cell wall of fungi. Since for TLPs, a relation exists 369 between the binding to these polysaccharides and their antifungal activity, the same 370 argumentation may be used for the xylanase inhibitors, which bind to $\beta$-glucans. Moreover,

371 recently, an extracellular peptidic bifunctional xylanase/aspartic protease inhibitor produced by 372 an extremophilic Bacillus sp. (ATBI) was shown to have antifungal properties (41). Fungal 373 growth inhibitions correlated well with the xylanase inhibition activities. This would mean that 374 the xylanase inhibitors may have a dual function in plant defence, i.e. the inhibition of the 375 xylanases produced by the intruders and the disturbance of the cell wall of the intruders. 


\section{Abbreviations}

377 WUAX: water-unextractable arabinoxylan; WEAX: water-extractable arabinoxylan; TAXI:

378 Triticum aestivum xylanase inhibitor; XIP: Xylanase inhibitor protein; TLXI: Thaumatin-like

379 xylanase inhibitor; PR: pathogenesis-related protein; TLP: Thaumatin-like protein

380

381 Acknowledgement

382 The autors wish to acknowledge Instituut voor de aanmoediging van Innovatie door wetenschap 383 en technologie in Vlaanderen (IWT, Brussels, Belgium) for financial support. Kurt Gebruers is a 384 postdoctoral fellow of the Fonds voor Wetenschappelijk Onderzoek-Vlaanderen (FWO385 Vlaanderen, Brussels, Belgium). 


\section{Literature cited}

387 1. Debyser, W.; Peumans, W. J.; Van Damme, E. J. M.; Delcour, J. A., Triticum aestivum 388 xylanase inhibitor (TAXI), a new class of enzyme inhibitor affecting breadmaking performance.

389 J. Cereal Sci. 1999, 30, 39-43.

390 2. McLauchlan, W. R.; Garcia-Conesa, M. T.; Williamson, G.; Roza, M.; Ravestein, P.; Maat, J., 391 A novel class of protein from wheat which inhibits xylanases. Biochem. J. 1999, 338, 441-446.

392 3. Fierens, E.; Rombouts, S.; Gebruers, K.; Goesaert, H.; Brijs, K.; Beaugrand, J.; Volckaert, G.;

393 Van Campenhout, S.; Proost, P.; Courtin, C. M.; Delcour, J. A., TLXI, a novel type of xylanase 394 inhibitor from wheat (Triticum aestivum) belonging to the thaumatin family. Biochem. J. 2007, $395403,583-591$.

396 4. Trogh, I.; Soerensen, J. F.; Courtin, C. M.; Delcour, J. A., Impact of inhibition sensitivity on 397 endoxylanase functionality in wheat flour breadmaking. J. Agric. Food Chem. 2004, 52, 42963984302.

399 5. Gebruers, K.; Courtin, C. M.; Moers, K.; Noots, I.; Trogh, I.; Delcour, J. A., The bread400 making functionalities of two Aspergillus niger endoxylanases are strongly dictated by their 401 inhibitor sensitivities. Enzyme. Microb. Technol. 2005, 36, 417-425. 
6. Frederix, S. A.; Courtin, C. M.; Delcour, J. A., Substrate selectivity and inhibitor sensitivity

403 affect xylanase functionality in wheat flour gluten-starch separation. J. Cereal Sci. 2004, 40, 4140449.

405 7. Gebruers, K.; Brijs, K.; Courtin, C. M.; Fierens, K.; Goesaert, H.; Rabijns, A.; Raedschelders, 406 G.; Robben, J.; Sansen, S.; Soerensen, J. F.; Van Campenhout, S.; Delcour, J. A., Properties of 407 TAXI-type endoxylanase inhibitors. Biochim. Biophys. Acta 2004, 1696, 213-221.

408 8. Juge, N.; Payan, F.; Williamson, G., XIP-I, a xylanase inhibitor protein from wheat: a novel 409 protein function. Biochim. Biophys. Acta 2004, 1696, 203-211.

410 9. Beliën, T.; Van Campenhout, S.; Van Acker, M.; Volckaert, G., Cloning and characterization 411 of two endoxylanases from the cereal phytopathogen Fusarium graminearum and their inhibition 412 profile against endoxylanase inhibitors from wheat. Biochem. Biophys. Res. Commun. 2005, 327, $413 \quad 407-414$.

414 10. Igawa, T.; Ochiai-Fukuda, T.; Takahashi-Ando, N.; Ohsato, S.; Shibata, T.; Yamaguchi, I.; 415 Kimura, M., New TAXI-type xylanase inhibitor genes are inducible by pathogens and wounding 416 in hexaploid wheat. Plant Cell Physiol. 2004, 45, 1347-1360.

417 11. Igawa, T.; Tokai, T.; Kudo, T.; Yamaguchi, I.; Kimura, M., A wheat xylanase inhibitor gene, 418 Xip-I, but not Taxi-I, is significantly induced by biotic and abiotic signals that trigger plant 419 defense. Biosci. Biotechnol. Biochem. 2005, 69, 1058-1063. 
420 12. Van Loon, L. C.; Van Strien, E. A., The families of pathogenesis-related proteins, their 421 activities, and comparative analysis of PR-1 type proteins. Physiol. Mol. Plant Pathol. 1999, 55, $422 \quad 85-97$.

423 13. Zhang, Z.; Collinge, D. B.; Thordal-Christensen, H., Germin-like oxalate oxidase, a H2O2424 producing enzyme, accumulates in barley attacked by the powdery mildew fungus. Plant J. 425 1995, 8, 139-145.

426 14. Wei, Y.; Zhang, Z.; Andersen, C. H.; Schmelzer, E.; Gregersen, P. L.; Collinge, D. B.; 427 Smedegaard-Petersen, V.; Thordal-Christensen, H., An epidermis/papilla-specific oxalate 428 oxidase-like protein in the defence response of barley attacked by the powdery mildew fungus. 429 Plant Mol. Biol. 1998, 36, 101-112.

430 15. Sancho, A. I.; Faulds, C. B.; Svensson, B.; Bartolome, B.; Williamson, G.; Juge, N., Cross431 inhibitory activity of cereal protein inhibitors against alpha-amylases and xylanases. Biochim. 432 Biophys. Acta 2003, 1650, 136-144.

433 16. Rouau, X.; Daviet, S.; Tahir, T.; Cherel, B.; Saulnier, L., Effect of the proteinaceous wheat 434 xylanase inhibitor XIP-I on the performances of an Aspergillus niger xylanase in breadmaking. 435 J. Sci. Food Agric. 2006, 86, 1604-1609.

436 17. Courtin, C. M.; Delcour, J. A., Arabinoxylans and endoxylanases in wheat flour bread437 making. J. Cereal Sci. 2002, 35, 225-243. 
439 19. Chen, W. P.; Chen, P. D.; Liu, D. J.; Kynast, R.; Friebe, B.; Velazhahan, R.; Muthukrishnan,

440 S.; Gill, B. S., Development of wheat scab symptoms is delayed in transgenic wheat plants that 441 constitutively express a rice thaumatin-like protein gene. Theor. Appl. Genet. 1999, 99, 755-760.

442 20. Datta, K.; Velazhahan, R.; Oliva, N.; Ona, I.; Mew, T.; Khush, G. S.; Muthukrishnan, S.;

443 Datta, S. K., Over-expression of the cloned rice thaumatin-like protein (PR-5) gene in transgenic 444 rice plants enhances environmental friendly resistance to Rhizoctonia solani causing sheath 445 blight disease. Theor. Appl. Genet. 1999, 98, 1138-1145.

446 21. Vigers, A. J.; Wiedemann, S.; Roberts, W. K.; Legrand, M.; Selitrennikoff, C. P.; Fritig, B., 447 Thaumatin-like pathogenesis-related proteins are antifungal. Plant Sci. 1992, 83, 155-161.

448 22. Trudel, J.; Grenier, J.; Potvin, C.; Asselin, A., Several thaumatin-like proteins bind to beta449 1,3-glucans. Plant Physiol. 1998, 118, 1431-1438.

450 23. Grenier, J.; Potvin, C.; Trudel, J.; Asselin, A., Some thaumatin-like proteins hydrolyse 451 polymeric beta-1,3-glucans. Plant J. 1999, 19, 473-480.

452 24. Osmond, R. I. W.; Hrmova, M.; Fontaine, F.; Imberty, A.; Fincher, G. B., Binding 453 interactions between barley thaumatin-like proteins and (1,3)-beta-D-glucans - Kinetics, 454 specificity, structural analysis and biological implications. Eur. J. Biochem. 2001, 268, 41904554199. 
456 25. Menu-Bouaouiche, L.; Vriet, C.; Peumans, W. J.; Barre, A.; Van Damme, E. J. M.; Rouge, 457 P., A molecular basis for the endo-beta 1,3-glucanase activity of the thaumatin-like proteins from 458 edible fruits. Biochimie 2003, 85, 123-131.

459 26. He, L.; Bickerstaff, G. F.; Paterson, A.; Buswell, J. A., Purification and partial 460 characterization of 2 xylanases that differ in hydrolysis of soluble and insoluble xylan fractions. 461 Enzyme. Microb. Technol. 1993, 15, 13-18.

462 27. Moers, K.; Celus, I.; Brijs, K.; Courtin, C. M.; Delcour, J. A., Endoxylanase substrate 463 selectivity determines degradation of wheat water-extractable and water-unextractable 464 arabinoxylan. Carbohydr. Res. 2005, 340, 1319-1327.

465 28. Gebruers, K.; Debyser, W.; Goesaert, H.; Proost, P.; Van Damme, J.; Delcour, J. A., Triticum 466 aestivum L. endoxylanase inhibitor (TAXI) consists of two inhibitors, TAXI I and TAXI II, with 467 different specificities. Biochem. J. 2001, 353, 239-244.

468 29. Bradford, M. M., Rapid and sensitive method for quantitation of microgram quantities of 469 protein utilizing principle of protein-dye binding. Anal. Biochem. 1976, 72, 248-254.

470 30. Biely, P.; Markovic, O.; Mislovicova, D., Sensitive detection of endo-1,4-beta-glucanases 471 and endo-1,4-beta-xylanases in gels. Anal. Biochem. 1985, 144, 147-151. 
472 31. Schwarz, W. H.; Bronnenmeier, K.; Grabnitz, F.; Staudenbauer, W. L., Activity staining of 473 cellulases in polyacrylamide gels containing mixed linkage beta-glucans. Anal. Biochem. 1987, $474 \quad 164,72-77$.

475 32. Royer, J. C.; Nakas, J. P., Simple, sensitive zymogram technique for detection of xylanase 476 activity in polyacrylamide gels. Appl. Environ. Microbiol. 1990, 56, 1516-1517.

477 33. Jue, C. K.; Lipke, P. N., Determination of reducing sugars in the nanomole range with 478 tetrazolium blue. J. Biochem. Biophys. Methods 1985, 11, 109-115.

479 34. Terwisscha van Scheltinga, A. C.; Hennig, M.; Dikstra, B. W., The 1.8 A resolution structure 480 of hevamine, a plant chitinase/lysozyme and analysis of the conserved sequence and structure of 481 motifs of glycosyl hydrolase family 18. J. Mol. Biol. 1996, 262, 243-257.

482 35. Perrakis, A.; Tews, I.; Dauter, Z.; Oppenheim, A. O.; Chet, I.; Wilson, K. S.; Vorgias, C. E., 483 Crystal structure of a bacterial chitinase at 2.3 A resolution. Structure 1994, 2, 1169-1180.

484 36. Payan, F.; Flatman, R.; Porciero, S.; Williamson, G.; Juge, N.; Roussel, A., Structural 485 analysis of xylanase inhibitor protein I (XIP-I), a proteinaceous xylanase inhibitor from wheat 486 (Triticum aestivum, var. Soisson). Biochem. J. 2003, 372, 399-405.

487 37. Reiss, E.; Schlesier, B.; Brandt, W., cDNA sequences, MALDI-TOF analyses, and molecular 488 modelling of barley PR-5 proteins. Phytochemistry 2006, 67, 1856-1864. 
489 38. Fierens, K.; Brijs, K.; Courtin, C. M.; Gebruers, K.; Goesaert, H.; Raedschelders, G.;

490 Robben, J.; Van Campenhout, S.; Volckaert, G.; Delcour, J. A., Molecular identification of 491 wheat endoxylanase inhibitor TAXI-I, member of a new class of plant proteins. FEBS Lett. 2003, $492540,259-263$.

493 39. Sibbesen, O; Soerensen, J. F. Enzyme. WO01/66711A1, 2001.

494 40. Rombouts, S. Molecular and mutational analysis of a wheat thaumatin-like xylanase inhibitor 495 (TLXI). Katholieke Universiteit, Leuven, Belgium, 2007.

496 41. Dash, C.; Ahmad, A.; Nath, D.; Rao, M., Novel bifunctional inhibitor of xylanase and 497 aspartic protease: Implications for inhibition of fungal growth. Antimicrob. Agents Chemother. $498 \quad 2001,45,2008-2017$.

499 


\section{Figure captions}

501 Figure 1: SDS-PAGE patterns of TAXI, XIP and TLXI after incubation with insoluble 502 (arabino)xylans. The first lane shows inhibitor control incubated in the absence of 503 polysaccharide $(\mathrm{C})$. The next lanes correspond to supernatant $(\mathrm{SN})$ and precipitate $(\mathrm{P})$ of wheat

504 WUAX, oat spelt arabinoxylan and birchwood xylan after preincubation with inhibitor and 505 centrifugation. The gels were run under reducing conditions and were silver-stained. On the right 506 side of the gels the MM at which the proteins were visible is indicated.

508 Figure 2: Inactivation of TAXI, XIP and TLXI by interaction with insoluble (arabino)xylans:

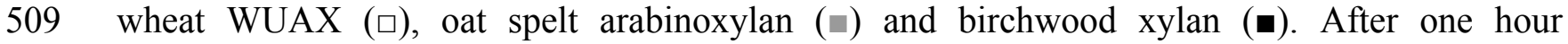
510 incubation of inhibitor with insoluble (arabino)xylans at $25{ }^{\circ} \mathrm{C}$ and $\mathrm{pH} 5.0$, the percentage of 511 bound inhibitor was estimated by comparing the inhibition activity in the supernatant of the 512 polysaccharide-inhibitor mixture to the inhibition activity of a control sample.

514 Figure 3: Typical enzymatic hydrolyses of soluble (arabino)xylans by a T. longibrachiatum (Xyn 515 I) xylanase for samples in which no inhibitor was added ( $\square$ ), in which xylanase and inhibitor 516 were preincubated $(\diamond)$ and in which inhibitor and substrate were preincubated $(\boldsymbol{\Delta})$. The 517 represented progress curves were plotted with XIP and wheat WEAX.

519 Figure 4: Inactivation of TAXI, XIP and TLXI by interaction with soluble (arabino)xylans:

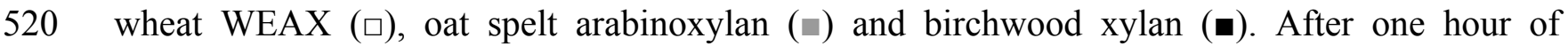
521 preincubation of inhibitor with soluble (arabino)xylans at $25{ }^{\circ} \mathrm{C}$ and $\mathrm{pH} 5.0$, the percentage of 
522 bound inhibitor was estimated by comparing the inhibition activity in this mixture to the activity

523 of inhibitor preincubated with xylanase instead of (arabino)xylan.

525 Figure 5: SDS-PAGE patterns of TAXI, XIP and TLXI after incubation with insoluble glucans.

526 The first lane shows the control inhibitor incubated in absence of glucans (C). The next lanes

527 correspond to supernatant $(\mathrm{SN})$ and precipitate $(\mathrm{P})$ of paramylon, oat $\beta$-glucan, zymosan, 528 cellulose and pustulan, after incubation with inhibitor and centrifugation. The gels were run 529 under reducing conditions and were silver-stained.

531 Figure 6: Inactivation of TAXI, XIP and TLXI by interaction with insoluble glucans: Paramylon

532 ( $\square$ ), oat $\beta$-glucan ( $\square$ ), zymosan ( $\square)$, cellulose $(\square)$ and pustulan ( $\square)$. After one hour of 533 preincubation of inhibitor with insoluble glucans at $25^{\circ} \mathrm{C}$ and $\mathrm{pH} 5.0$, the percentage of bound 534 inhibitor was estimated by comparing the inhibition activity in the supernatant of the 535 polysaccharide-inhibitor mixture to the inhibition activity of a control sample.

537 Figure 7: Inactivation of TAXI, XIP and TLXI by interaction with soluble glucans: CM538 pachyman ( $\square$ ), laminarin $(\square)$ and pullulan ( $(\mathbf{)})$. After one hour of preincubation of inhibitor with 539 soluble glucans at $25{ }^{\circ} \mathrm{C}$ and $\mathrm{pH} 5.0$, the percentage of bound inhibitor was estimated by 540 comparing the inhibition activity in the supernatant of the polysaccharide-inhibitor mixture to the 541 activity of inhibitor incubated similarly without polysaccharide. 


\section{Tables}

543

544 Table 1: Structures, sources and solubilities of different glucans

\begin{tabular}{cccc}
\hline Polysaccharides & Structures & Sources & Solubilities \\
\hline Paramylon & $\beta-(1,3)$-glucan & Euglena gracilis & Insoluble \\
$\beta$-glucan & $\beta-(1,3)(1,4)$-glucan & Graminaceae (Oats) & Insoluble \\
Zymosan & Complex cell wall & Saccharomyces & Insoluble \\
& polysaccharide containing & cerevisiae & \\
& $\beta-(1,3)$ - and $\beta$ - $(1,6)$-glucan & & \\
Cellulose & $\beta-(1,4)$-glucan & Plants & Insoluble \\
Pustulan & $\beta-(1,6)$-glucan & Umbilicaria papulosa & Insoluble \\
CM-Pachyman & $\beta-(1,3)$-glucan & Poria coccos & Soluble \\
Laminarin & $\beta$-(1,3)-glucan, contains & Laminaria digitata & Soluble \\
& some $\beta-(1,6)$-linkages & & \\
Pullulan & $\alpha-(1,4)(1,6)$-glucan & Aureobasidium pullulans & Soluble \\
\hline
\end{tabular}


545 Figures

546

$547 \quad$ Figure 1

548

549

550

551

552

553

554

555

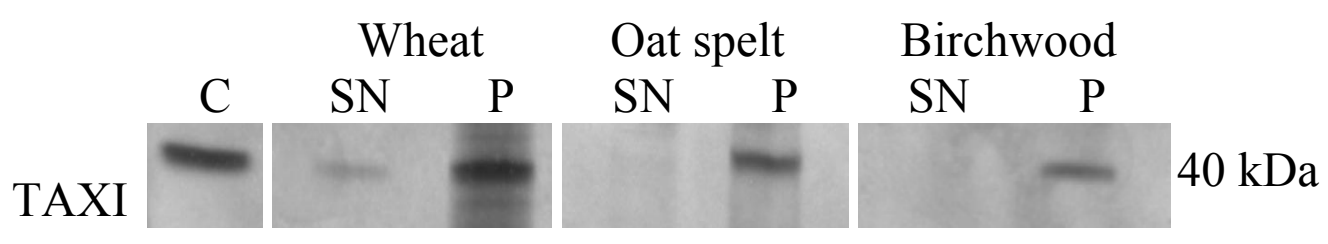

556
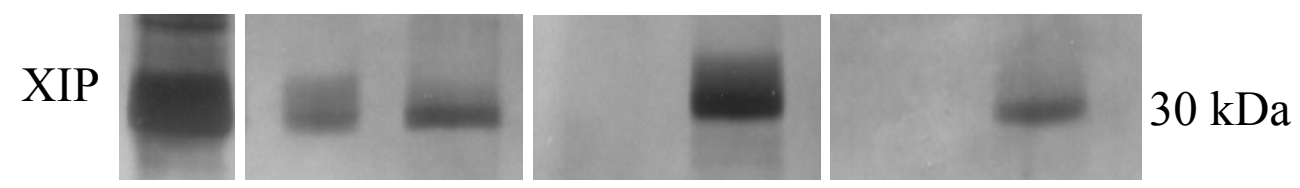

557

TLXI
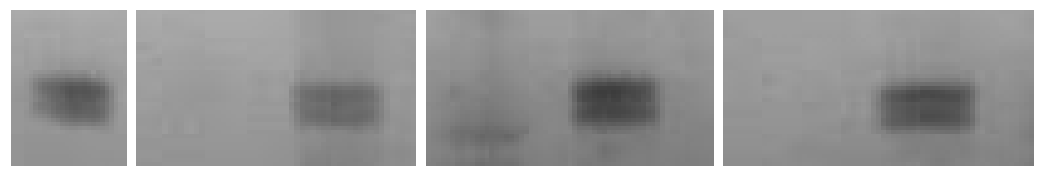

$18 \mathrm{kDa}$ 
558 Figure 2

559

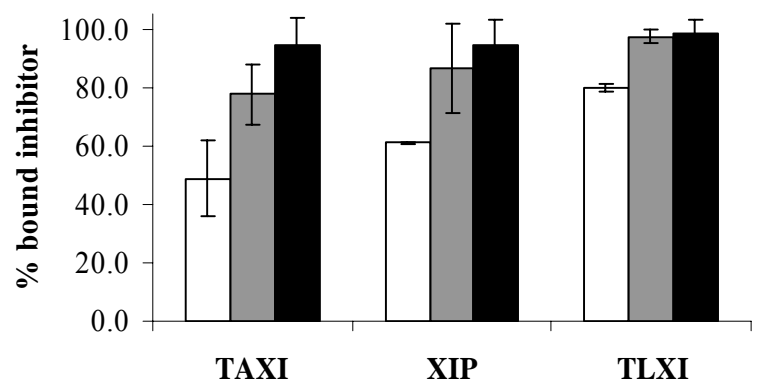

560

561 
562 Figure 3

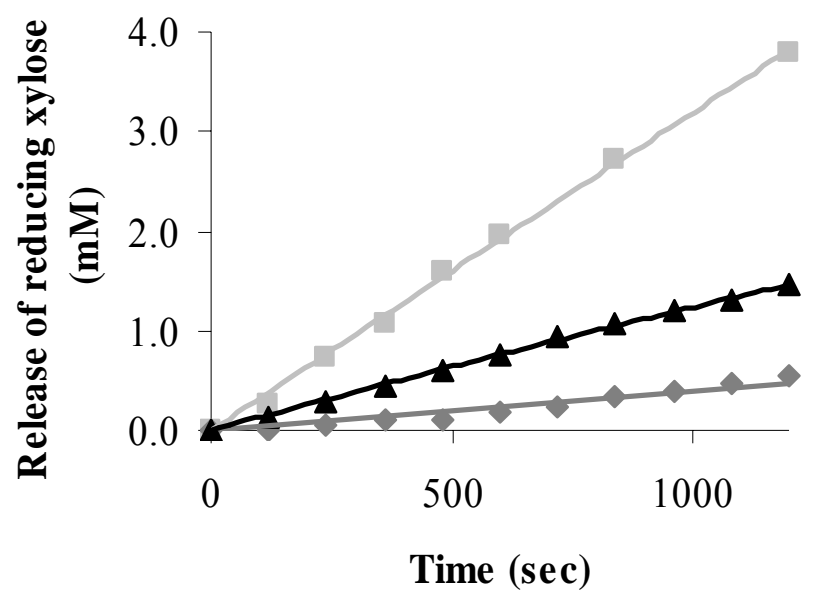

563 
564 Figure 4

565

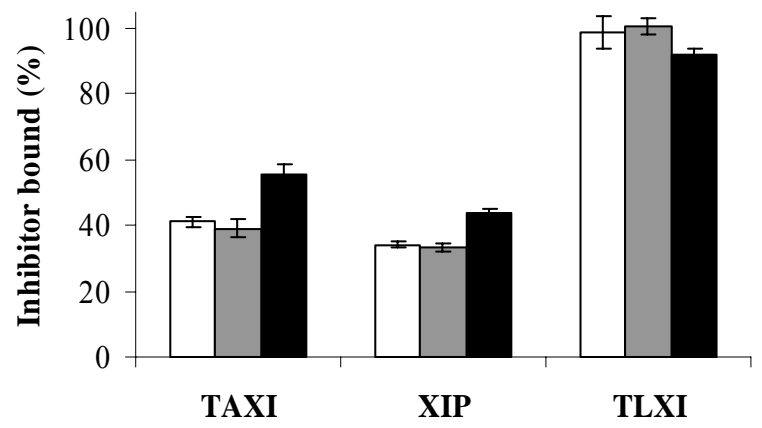

566

567 
568 Figure 5

569

570

571

TAXI

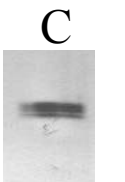

Paramylon Oat-glucan

Zymosan Cellulose

Pustulan

\begin{tabular}{|c|c|c|}
\hline $\mathrm{C}$ & SN $\mathrm{P}$ & SN $\mathrm{P}$ \\
\hline TAXI $=$ & $=$ & - \\
\hline
\end{tabular}

SN P

SN P SN P
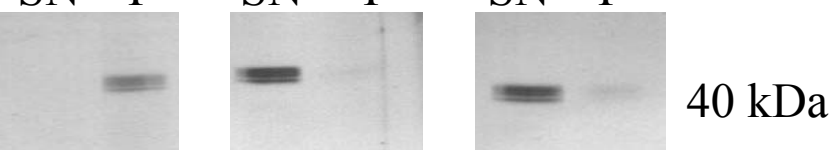

572

$$
\text { XIP }
$$
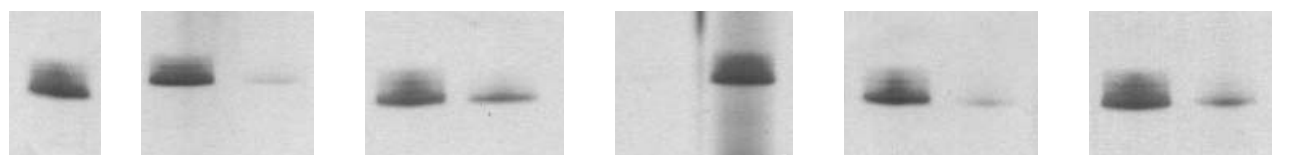

$30 \mathrm{kDa}$

TLXI
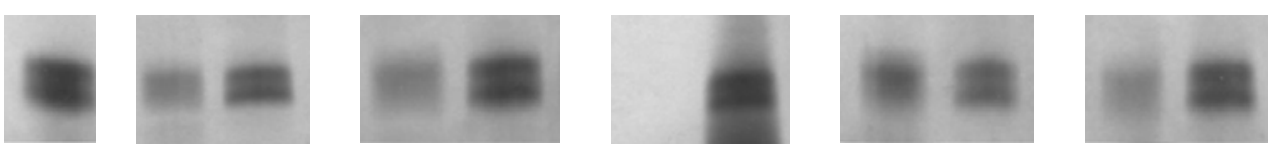

$18 \mathrm{kDa}$

574

575 
$576 \quad$ Figure 6



577

578 
$579 \quad$ Figure 7

580

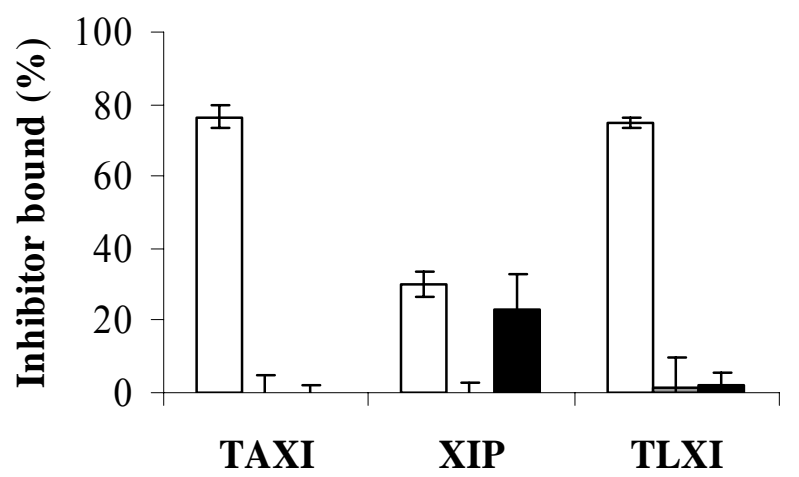

581

582

583

584

585

586

587

588

589

590

591 